Original Research Paper

\title{
Flexible Stem Trabecular Prostheses
}

\author{
${ }^{1}$ Raffaella Aversa, ${ }^{2}$ Florian Ion T. Petrescu, ${ }^{3}$ Relly Victoria V. Petrescu and ${ }^{1}$ Antonio Apicella \\ ${ }^{1}$ Advanced Materials Lab, \\ Department of Architecture and Industrial Design, Second University of Naples, Aversa (CE) Italy \\ ${ }^{2}$ Department of Theory of Mechanisms and Robots, Bucharest Polytechnic University, Bucharest, Romania \\ ${ }^{3}$ Department of Transport, Traffic and Logistics, Bucharest Polytechnic University, Bucharest, Romania
}

Article history
Received: 01-12-2016
Revised: 08-12-2016
Accepted: 21-12-2016
Corresponding Author:
Florian Ion T. Petrescu
Department of Theory of
Mechanisms and Robots,
Bucharest Polytechnic
University, Bucharest,
Romania
Email: scipub02@gmail.com

\begin{abstract}
The complex biomechanics and morphology of the femur proximal epiphysis are presented. This specific region in human femur is characterized by a high flexibility compared to that of other primates, since evolved lighter and longer due to the human verticalposition and more balanced loading. The nature and fine morphology of the femur head and its structural behavior have been investigated. Isotropic and orthotropic trabecular structures, which are not present in other primates, have been associated to compression and tensioned areas of the femur head. These isotropic/orthotropic trabecularmorphologies and allocationsgovern the stress and strains distribution in the overall proximal femur region. Useof femur proper biofidel modeling while enabling the explanationof physiological stress distribution elucidates the critical mechanical role of the trabecular bone that should be accounted in the design new innovative more "biologic" prosthetic system.
\end{abstract}

Keywords: Trabecular Lattice, Biomimetic, Biomechanics, Trabecular Prostheses

\section{Introduction}

Today used orthopedic prostheses are mainly made of metals and ceramics materials with outstanding strengthand rigidity properties but high physiological invasiveness. These systems, while guarantying biomechanical functional tenure, they often strongly interfere with human bone physiology.

This invasiveness is particularly evident for hip joint replacementsusing rigid full metal prostheses. Human bipedal gait and vertical posture have lead to unique and considerable evolution and adaptations of the femur, pelvis, hip and lumbar vertebrae osseous systems.

The complex evolution of the trabecular bone positioning and morphology in human proximal femur has been primarily determined by stress patterns where bone was retained only in the areas where it underwent sufficient straining mechanical stimulus and it was lost when not (Lovejoy, 1988; 2005; Lovejoy et al., 2002).

Therefore, clinical efficiency and long-term reliability of hip joint prosthesization needsa more deep understanding of the biomechanical invasiveness of the current restorative replacements.These products, which are made from traditional technologies such as melting and mechanical machining, do not hold flexible design solutions that permit good biomechanical bone integration in the biologically complex femur bone structure. Moreover, these traditional solutionsare not adequate for younger patients who have high life expectations and then high prostheses requirements in terms of duration and biomechanical osseointegration.

New generation of prostheses with better biomechanical osseointegration with the living bone are then needed.

In particular, additive technologies using Titanium or Cobalt Chrome alloys, due to their process eclecticism in generating high strength complex trabecular structures, can represent the future new generation of flexible trabecular bio-prosthetic components productions.

Studies on prostheses biomimetics involving these innovative fabrication processes (Annunziata et al., 2006; Apicella et al., 2010; Aversa et al., 2009; 2016) have opened to the definition of new design criteria for more biomechanically compatible prostheses production. Figure 1 illustrates the biomimetic approach using in silico, in vitro and in vivo validation steps for biofidel bone modeling.

The biofidelmodels of the mandible and of the femurhave been presented in previous publications 
(Apicella et al., 2010; Gramanzini et al., 2016; Perillo et al., 2010; Sorrentino et al., 2007; 2009).

The Authors, starting from these studies, have investigated the still not fully exploited potential of additive manufacturingtechnologies. Moreover,the advances of our biomimetic design procedures, whichhave already enabled us to conceptually develop new biomimetic dental implant (Aversa et al., 2009) and new trabecular prostheses, couldlead to new prosthetic systems that better mimic the femur biomechanical behavior (Aversa et al., 2016).

Human femur has an internal light trabecular structure that, through its evolutionary optimization of the mass and morphology of the cortical and trabecular bone types (Walker and Lovejoy, 1985; Bruno et al., 1999; Oh and Harris, 1978; Tamar and Hashin, 1980), has been able to develop mechanical properties that can bear high external stresses (Ashman et al., 1984; Dalstra et al., 1993).

Human bipedal gait and vertical posture have lead to unique and considerable adaptations of the femur, pelvis, hip and lumbar vertebrae osseous systems. In particular, the hip joint acquired a much more extended position. The human femur, which is principally loaded in the vertical position, has evolved lighter and longer than for other primates where it is loaded horizontally.

This morphological evolution was due because femoral neck of the limb in vertical stand is loaded as a cantilevered beam and it can be then related to the smaller bending moments that are generated in humans.

The evolutionary path of the human hip have been extensively reviewed (Lovejoy, 2005) to explain the uncommon cortico-trabecular structure of the proximal femur, which presents definitely thickened cortical bone only in specific locations (Fig. 2 right).

In particular, as reported by Lovejoy (1988), the femur head thickens the cortex only near its lower portion while it exhibits a practically complete cortex absence in its upper portion as reported in Fig. 2 right).

Conversely, very high cortical bone is apposed in the medial diaphysis where flexural stresses are predominant (Fig. 2). The complexity of the human proximal femur morphological positioning of the trabecular bone in the proximal femur Fig. 2 and 3) is primarily determined by rearrangements where bone is retained only in the regions where it reaches a sufficient straining stimulus and, conversely, it is lost when not (Sims and Martin, 2014).

Gluteus abductors reduce extension strain in the upper part of the femoral neck to such an extent that trabeculae coalesced by progressive loss of arcing trabeculae forming the Ward's Triangle which is less radiopaque than in other primates.

The morphology of the trabecular bone in the region of the proximal epiphysis (femur head) depends on the stress state. Figure 3(left) shows the epiphysis radiographicimage where a clearly visible the trabecular orientation patterns for compressed (blue lines) and tensioned regions (red lines). The morphologies of the trabeculae in these two regions are completely different (Fig. 3, right side): Spherical pores are observed for compression while they appear elongated in the tensioned regions. The mechanical behavior of these two structures is completely different: Isotropic for the spherical trabeculae and orthotropic (directional) for the elongated trabeculae.

The solid models of the trabecular structures obtained from Micro Computer Tomography (Micro CT) in compressed (Fig. 4a) and tensioned (Fig. 4c) regions clarify the origin of this different mechanical behavior. The structure that is repeating in the trabecular bone of the compressed regions are constituted by repeating tetrahedral/octahedral units (Fig. 4b). The more complex structure of the tensioned trabeculae (Fig. 4c) is characterized by a different orientation and distribution in transversal (Fig. 4d) and longitudinal (Fig. 4e) sections. This distribution, which is more continuous and massive in the longitudinal section, leads to a greater strength and rigidity of the bone in the direction of the trabeculae orientation (Fig. 4e) than in the transvers direction (Fig. 4d).

Total hip replacements inyounger patients (less than 65) should meet higher life span expectation thatneeds anextendedfunctional permanency of the prosthesis overcoming the actual 15 years. A more biological and biomimetic prosthesis combining the needed strength withmodulated stiffness that match that of the bone has to be developed. The prosthesis that replacesthe resected portions of the femur head, should be expected to ensure an "equivalent stiffness", which cab be obtained by combining trabecular morphology andprosthesis shape, that matches that of the missing part and inducing equivalent deformation of the residual osseous region. The higher mechanical compatibility allowing better bio- and osseointegration will increase its service lifespan avoiding implant loosening and any additional surgicalrevision.

Figure 5 shows the adaptive properties of the bone undergoing coupling between bone formation and bone reabsorption. This process relates to bone-forming activity in which the osteocytes reabsorption by osteoclasts and the renewed generations of osteoblasts from precursors replaces in a dynamic equilibrium the bone. Coupling can be then considered a complex dynamic remodelling mechanism involving the interactions of different types of cells and controlling stimuli. Sinceremodelling of the bone occurs at many sites "asynchronously throughout the skeleton" (Sims and Martin 2014), control mechanisms comprising the mechanical stimulation are locally active (Fig. 6). The mechanical stimulation should be comprised between physiological strain levels 
(Fig. 6) between $50 \mu \varepsilon$ and $3000 \mu \varepsilon$ (Apicella et al., 2011; 2015; Aversa et al., 2009).

Osteoblasts under specific biochemical and mechanical stimulations mature and transform in osteocytes that mineralize the bone. The osteoclasts activity in not mechanically stimulated conditions following prosthesization could then induce bone reabsorption in this new mechanical equilibrium state.

The traditional full metal implants adopted today, which are characterized by stiffness much higher than those of bone where they are implanted, strongly alters the physiological local deformation leading to undesired bone reabsorption. The stiffness mismatching, in fact, leads to the low level of loading, which is know as stress shielding, that reduces the physiological strain needed for healthy bone growth (Frost, 1994; Weinans et al., 1992).

Conversely, acustomized metallic trabecular structure, which combines both strength and elasticity requirements, may produce a beneficial healthy growth physiological mechanical stimuluseven in the prosthesized bone.

The trabecular metal structure of the new prostheses could be achieved using Electron Beam (EB) sintered Titanium alloys. The EB Additive technology melts Titanium powders of about 50 microns forming thin layers one over the other.

Moreover, biological hybrid prostheses made using hybrid polymeric materials (Schiraldi et al., 2004) coatings may further favor the growth of the bone and prosthesis biointegration.

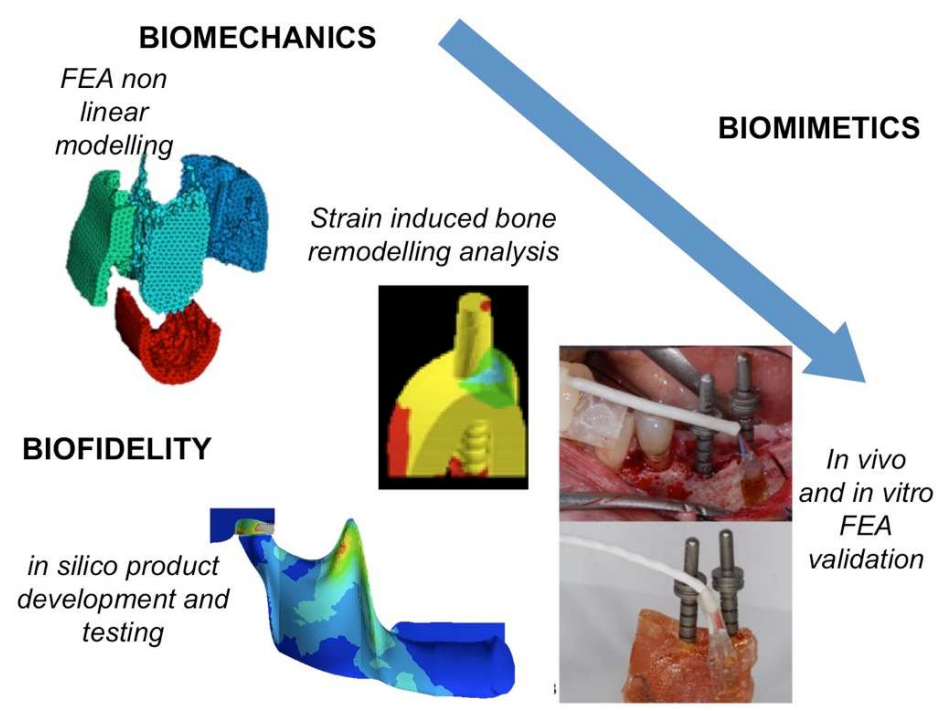

Fig. 1. Biomimetic approach from bone biomechanics (mandible case study with in silico, in vitro and in vivo validation steps)

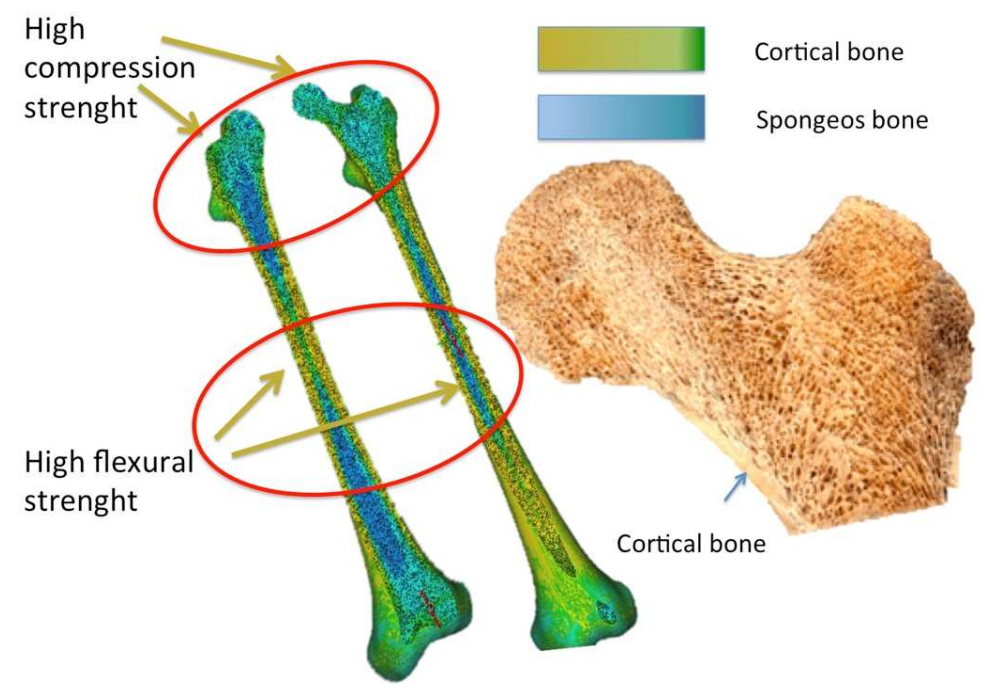

Fig. 2. Cortical and trabecular bone distribution in the entire femur (left) and internal complex patterns of the trabecular bone in the proximal epiphysis section (right) 


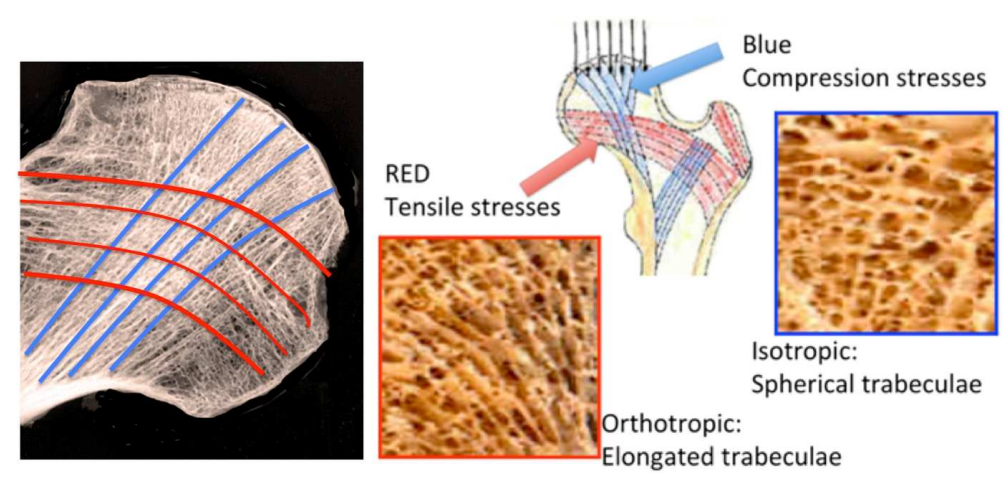

Fig. 3. Radiographic evidence of oriented trabecular structure in compression (blue lines) and tensioned (red lines) and trabeculae isotropic (compression) and orthotropic (tension) morphology
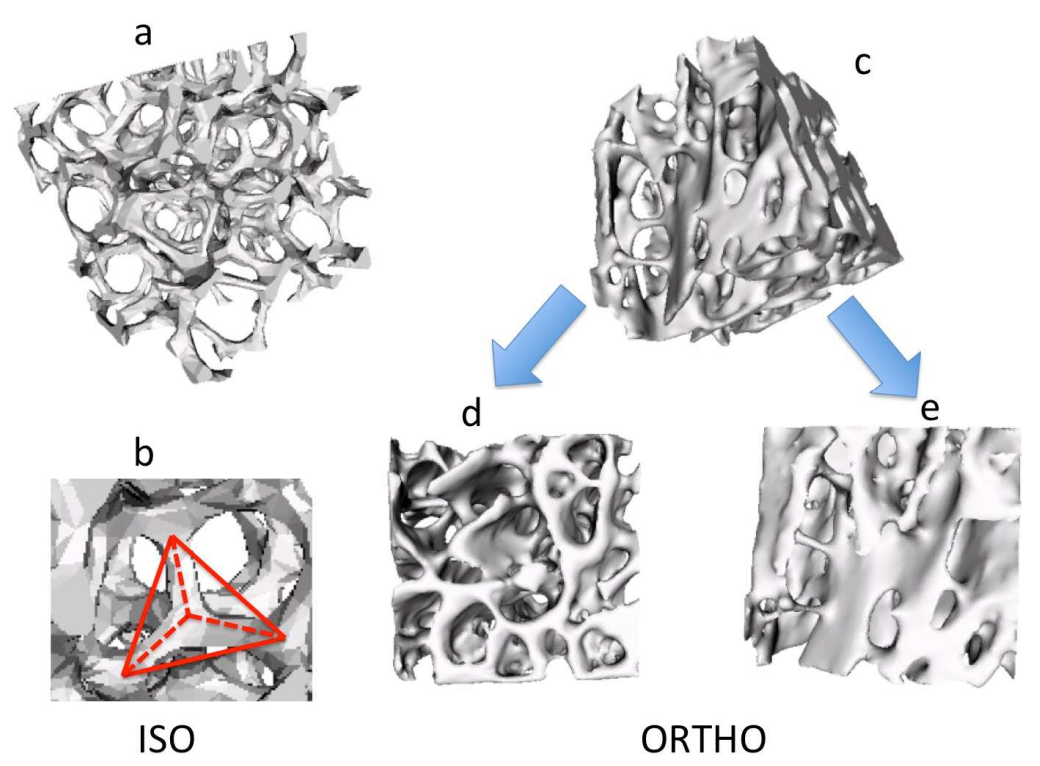

Fig. 4. Solid models of femur iso (left) and ortho (right) bone trabecular structures from Micro CT of femur

\section{Bone adaptive properties}

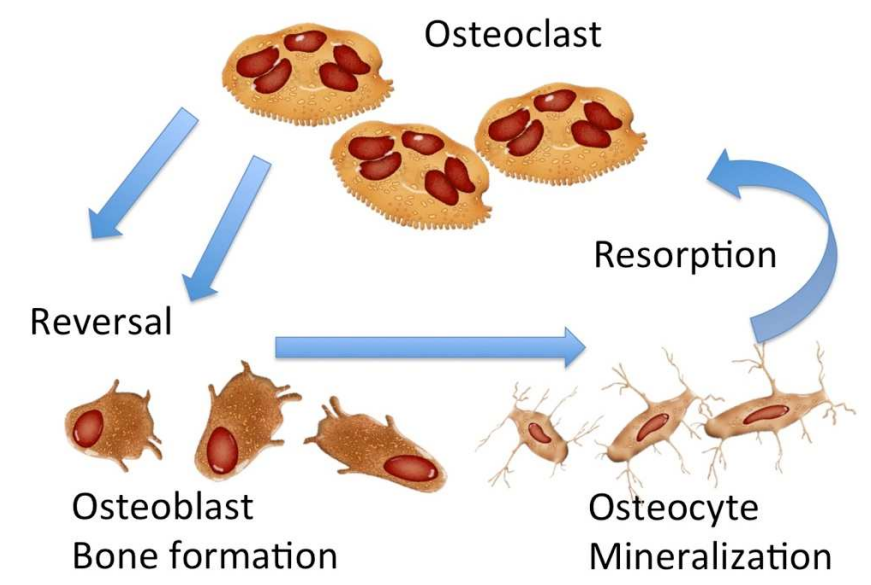

Fig. 5. Bone adaptive properties: Coupling between bone formation and bone reabsorption 


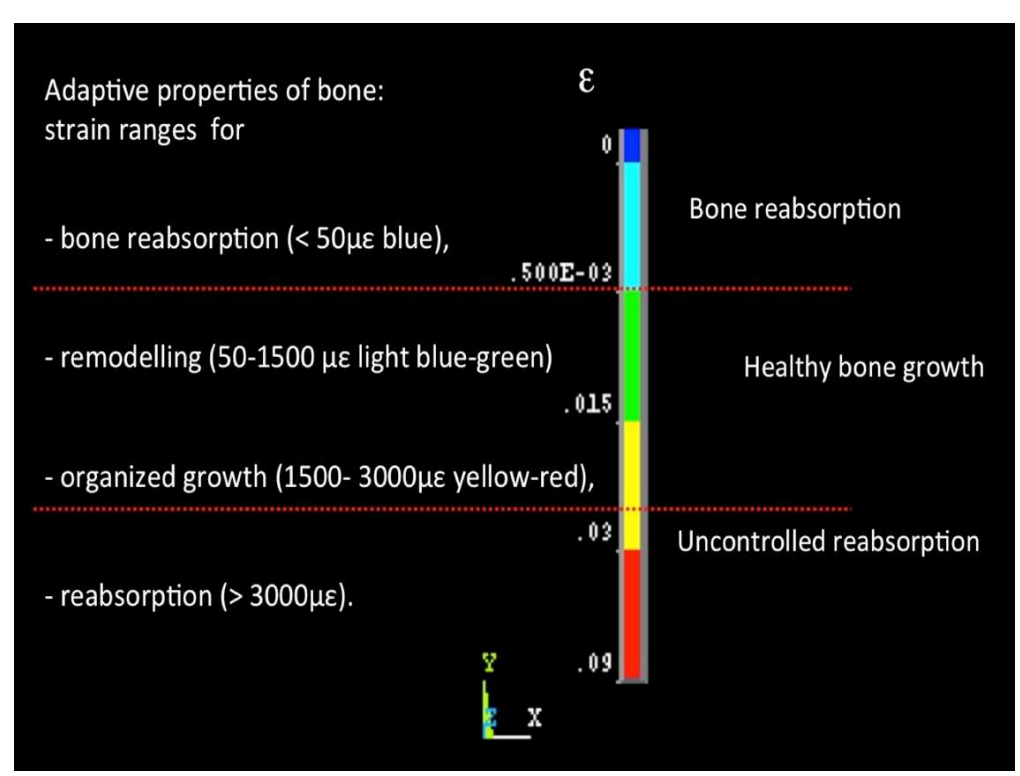

Fig. 6. Bone adaptive properties: Range of physiological strains for bone growth

The new hybrid bio-prosthesis could drastically reduce the stress shielding phenomena while providing an advantageous improvement of prosthesis lifetime compared to traditional solutions. The recovery of optimal joint functionality will lead to the improvement of the quality of life of the patient, who perceives significantly abating the risk of new surgical operation.

In this study we analyze the potential of the new additive technologies in the development of trabecular prostheses that better mimics the resected bone mechanical behavior that could lead to a new generation of biomimetic prostheses.

Finite Element Models have been presented in previous works where the influence of the morphology of femur and mandible structureshas been evaluated (Aversa et al., 2016, 2009; Apicella et al., 2010; Beaupre and Hayes, 1985; Reilly and Burstein, 1974; 1975).

\section{Methods and Discussion}

Medical Image Segmentation has been derived using the Mimics and 3Matic softwares (Materialise, Belgium) to process $\mathrm{CT}$ and micro CT medical imaging of a entire femur and its proximal epiphysis lead to the solid models reported infigures 2 and 4 and to the STL files of Fig. 7.

The results of tethraedric element material distribution are shown in Fig. 2. The yellow-green elements correspond to cortical bone while light and dark blues are relative the trabecular bone.

The same procedure was applied from Micro CT segmentation of a sectioned proximal epiphysis (Fig. 4) to the preparation of solid models of the trabecular bone types, which characterize the micro morphology of the femur head trabecular bone types.
Modeling of trabecular bone structures from Micro TAC segmentation was carried out on a section of a dry femur (Fig. 2 and 3). The trabecular bone of the femoral head, in fact, is characterized by different morphologies that are influenced by their of position. In particular, a structure characterized by asymmetric pores elongated in the direction of the lines of tension is evident in areas subject to tension while in the areas subject to compression the trabeculae follow symmetrical arched structure.

The objective of modeling these regions was aimed to identify the exact morphology and properties of the trabecular structures to mimic successive replication of equivalent structures obtained by additive technology (Fig. 7 and 8).

\section{Orthotropic Trabecular Bone (Fig. 7 Right Side)}

The CAD solid model and solid meshes of the asymmetric trabecular bone, which is expected to show mechanical properties of an orthotropic structure, have been obtained from MicroTac of a trabecular segment $4,5 \times 4,5 \times 4,5 \mathrm{~mm}$ processed with Materialise Mimics and 3 Matic software.

Detailed surface mesh (about 300,000 units) of the trabecular structure volume mesh (reduced size) of about 1200 solid elements (4 knots tetrahedrons elements) are reported in Fig. 7 (right side).

The apparent density of the trabecular bone, which has been evaluated as the ratio between the volume occupied by trabeculae $\left(36.5 \mathrm{~mm}^{3}\right)$ and the total volume occupied $\left(103.7 \mathrm{~mm}^{3}\right)$ is 0.665 grams per $\mathrm{cm}^{3}$ (this has been evaluated by considering a limiting density for the cortical bone of $1.9 \mathrm{~g} / \mathrm{cm}^{3}$ ). 


\section{Isotropic Trabecular Bone (Fig. 7 Left Side)}

Analogously, the CAD solid model and mesh of the symmetric trabecular bone, which is expected to show isotropic mechanical properties, have been obtained from MicroTac segmentation of a trabecular segment $16 \times 16 \times 16 \mathrm{~mm}$ processed with Materialise Mimics and 3Matic softwares.

From this surface CAD model it was generated the STL file and the solid model FEM with the operation of 3 matic volume mesh. The volume generated contains approximately 1900 tetrahedral elements and 31700 nodes. The bulk density of the isotropic trabecular bone, which has been derived from the ratio between the volume occupied by the trabeculae $\left(33,167 \mathrm{~mm}^{3}\right)$ and the overall occupied volume $\left(2340 \mathrm{~mm}^{3}\right)$ size with an apparent density of $0.070 \mathrm{~g} / \mathrm{cm}^{3}$ ).

The equivalent isotropic and orthotropic structures in Titanium will have apparent densities of 1.3 and $0.15 \mathrm{~g} / \mathrm{cm}^{3}$. The equivalent structures to be used in the EB additive sintering of $\mathrm{Ti}$ powder (Fig. 8) are illustrated in Fig. 9. Namely, an equivalent octahedral repeating unit has been chosen for the isotropic trabecular bone (Fig. 9 left side) and an octaedroid with the ratio between the three principal orthogonal directions 121 (Fig. 9 right side) has been chosen for the orthotropic trabecular structure.

\section{Variable Rigidity Ti Prostheses Design}

In order to reduce the rigidity of the prosthesis stem, a trabecular structure has been properly designed to match the specific rigidity of each diaphysis section interested by the prosthesization.

The proposed model of the hip proximal epiphysis follows the isostaticlines that characterizes the oriented trabecular systems (Kummer, 1986) and it is shown in Fig. 9 (lower side). Details of the trabecular prosthesis are given in Fig. 10.

The five regions of the prosthesis decreasing rigidities are contained in the red lines reported in the right low side of Fig. 10 along with isotension black lines.
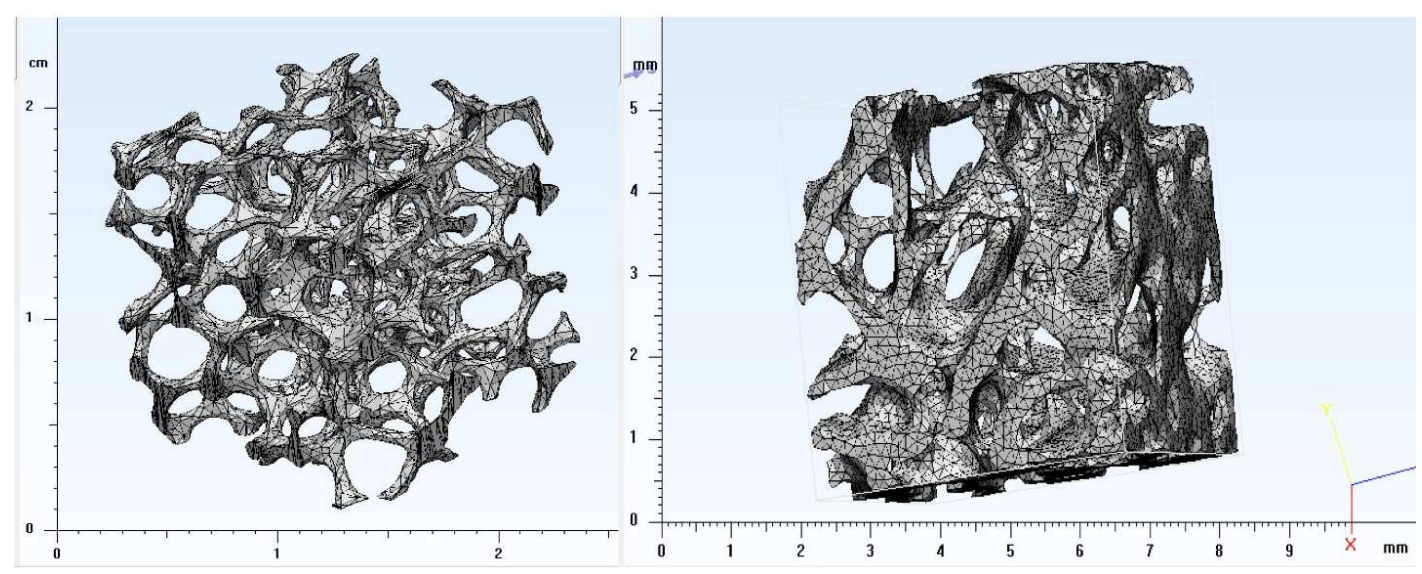

Fig. 7. Discretized solid models for STL and FEA files generation
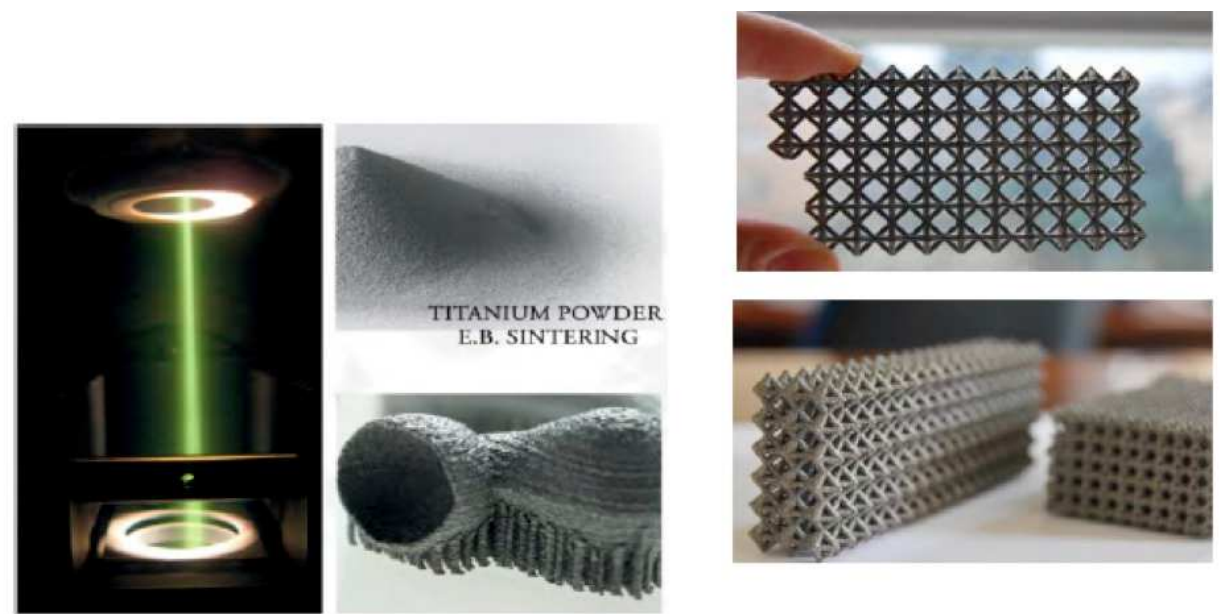

Fig. 8. Biomimetic trabecular prostheses design from Ti powders sintering by Electron Beam Manufacturing 


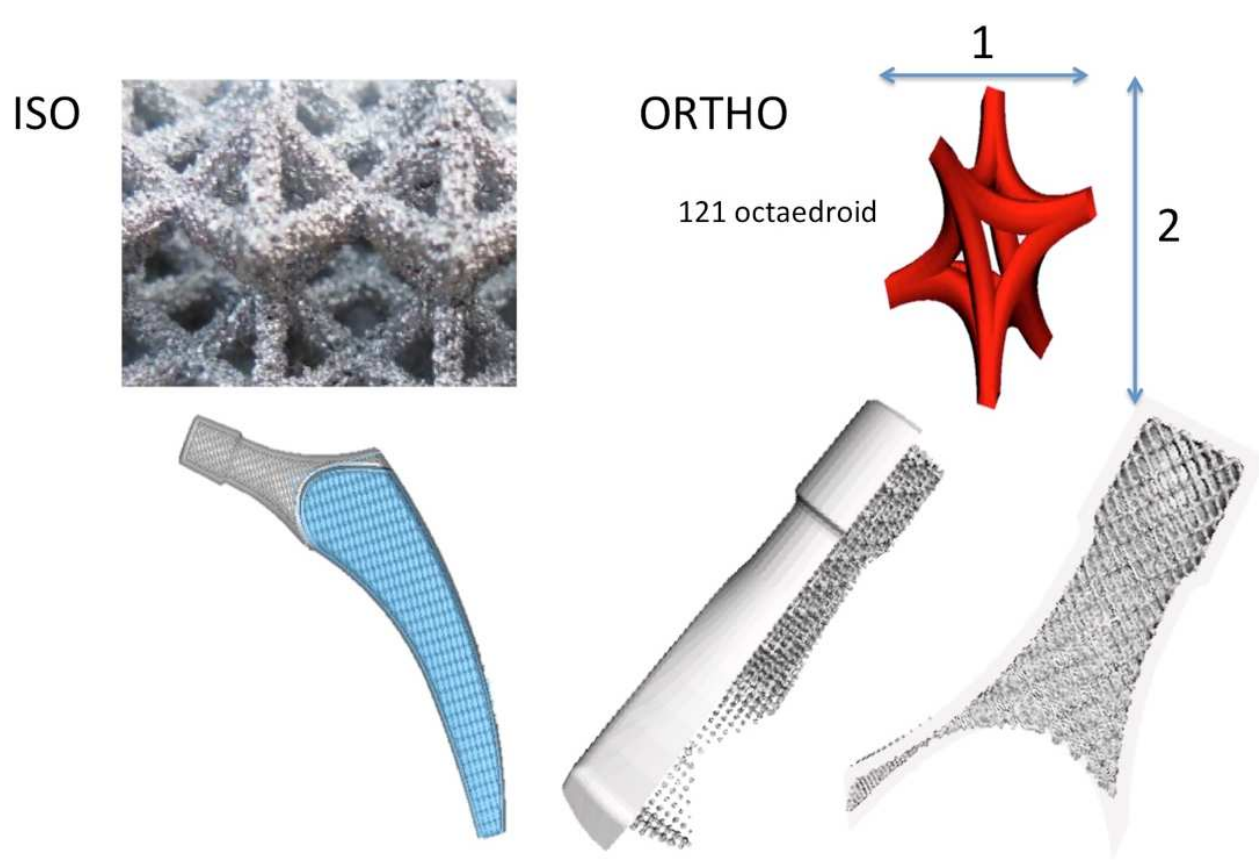

Fig.9. Trabecular prosthesis with iso (left, stem) and with ortho (right, head) structures.
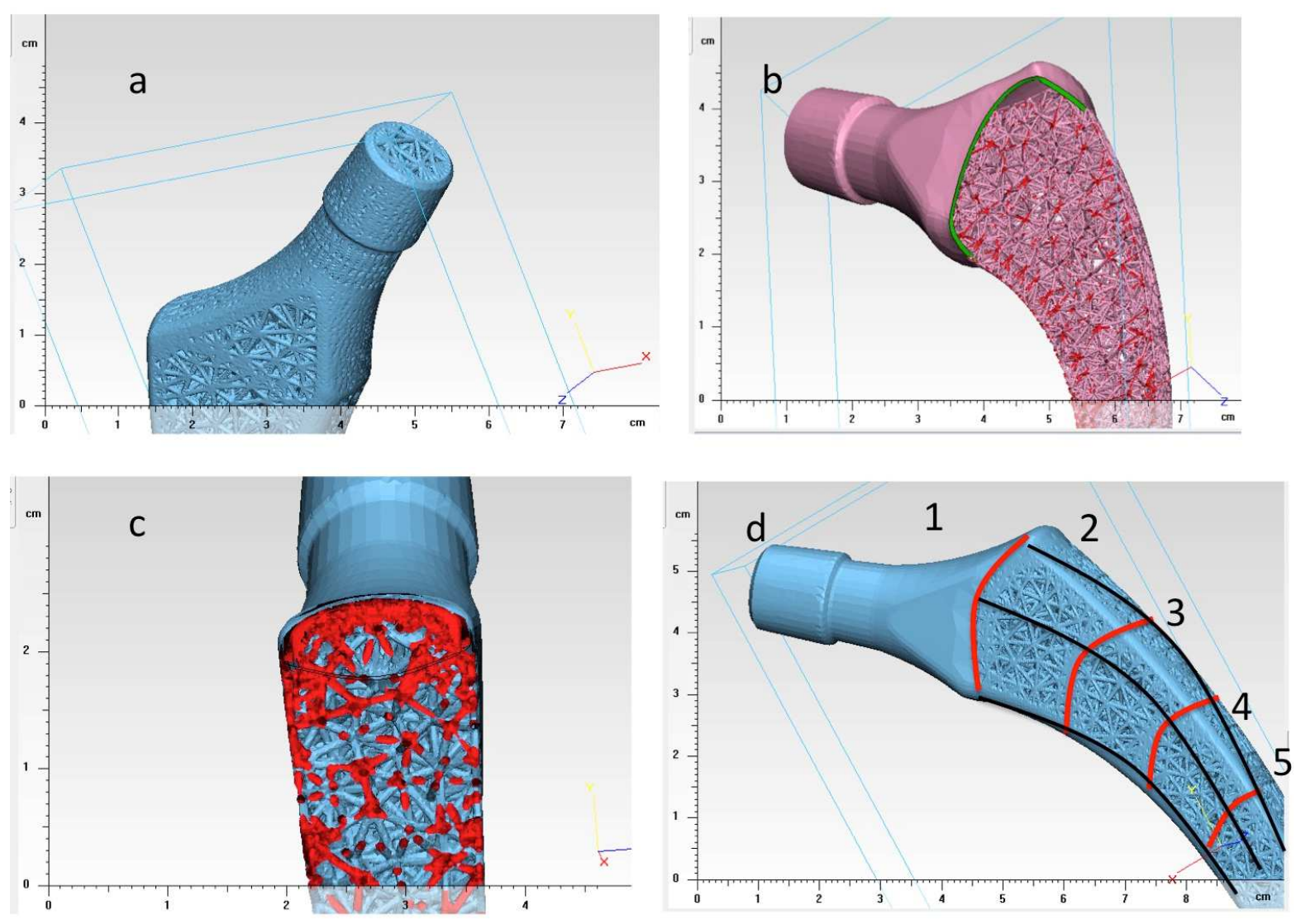

Fig.10. Flexible trabecular hip joint prosthesis with trabecular structure obtained by additive sintering of Ti powders. (a) Internal trabecular structure of the prosthesis, (b) Stem longitudinal section (c) Stem transversal section, (d) Isorigidity regions (red) and isotension lines (black) 
Table 1. Elastic moduli distribution in the isorigidity regions

\begin{tabular}{llllll}
\hline Region & 1 & 2 & 3 & 4 & 5 \\
\hline $\mathrm{E}, \mathrm{GPa}$ & 20.0 & 11.0 & 8.0 & 4.0 & 1.0 \\
\hline
\end{tabular}

These regions are characterized by different rigidities that progressively decrease from the highly rigid head (region 1 in Fig. 10) down to the lowerrigidity one (region 5 in Fig. 9).

The orientation and shape of the trabecular porosity of the prosthesis is evident in the details of the internal structure reported in the transverse and longitudinal sections reported in Fig. 10b and 10c.

Isorigidity trabecular structure obtained by $\mathrm{Ti}$ alloy powder sintering by Electron Beam is reported in Fig. 10d. The apparent elastic moduli of the trabecular structures are summarized in Table 1.

\section{Conclusion}

The proposed paper illustrates thenew concept of biomimetic prostheses obtained using trabecular structure in substitution of full metal implants. The currently used prosthesis while restoring the hip functionality, do not preserve the physiological beneficial strain distribution in the remaining bone surrounding the prosthesis. The new more biological and flexible prostheses are able to be completely biomimetic since they are mimicking and recovering the physiological strain distribution of the bone.

It could be expected that the new average life of these flexible prosthesis could overcome the limits of 10/15 years of the current prosthetic systems

This work has identified design criteria and manufacturing technologies to exploit the potential of new customized health care. The new class of implants may be extended to the orthopedics of the ankle, the hip and the knee.

The new flexible prosthetic system allows better functional recovery and bone regeneration due to their biomechanical activity.

Application of the product is surgical oncology is also possible in the restoring of removed bone section of primitive and metastatic tumors.

\section{Acknowledgement}

We acknowledge and thank MrTaher M. AbuLebdeh, Associate Prof at North Carolina A and T State Univesity, United States and MrMuftah H. El-Naas PhD MCIC FICCE QAFCO Chair Professor in Chemical Process Engineering Gas Processing Center College of Engineering Qatar University and MsShweta Agarwala, Senior Research Scientist at Singapore Center for 3D Printing Nanyang Technological University Singapore for their suggestions and comments.

\section{Funding Information}

This research was partially funded by Italian Ministry of University and Research with the project FIRB "Future in Research 2008",\# RBFR08T83J.

\section{Author's Contribution}

All the authors contributed equally to prepare, develop and carry out this work.

\section{Ethics}

This article is original. Author declares that are not ethical issues that may arise after the publication of this manuscript.

\section{References}

Annunziata, M., R. Aversa, A. Apicella, A. Annunziata and D. Apicella et al., 2006. In vitro biological response to a light-cured composite when used for cementation of composite inlays. Dental Mater., 22: $1081-1085$.

DOI: $10.1016 /$ j.dental.2005.08.009

Apicella, D., R. Aversa, E. Ferro, D. Ianniello and A. Apicella, 2010. The importance of cortical bone orthotropicity, maximum stiffness direction and thickness on the reliability of mandible numerical models. J. Biomed. Mater. Res. Part B Applied Biomater., 93: 150-163.

DOI: $10.1002 / \mathrm{jbm} . b .31569$

Apicella, D., M. Veltri, P. Balleri, A. Apicella and M. Ferrari, 2011. Influence of abutment material on the fracture strength and failure modes of abutmentfixture assemblies when loaded in a bio-faithful simulation. Clin. Oral Implants Res., 22: 182-188. DOI: $10.1111 /$ j.1600-0501.2010.01979.x

Apicella, D., R. Aversa, M. Tatullo, M. Simeone and S. Sayed et al., 2015. Restoration modalities of fractured central maxillary incisors: A multi-levels validated finite elements analysis with in vivo strain measurements. Dental Mater., 31: e289-e305. DOI:10.1016/j.dental.2015.09.016

Ashman, R.B., S.C. Cowin, V.W.C. Buskirk and J.C. Rice, 1984. A continuous wave technique for the measurement of the elastic properties of cortical bone. J. Biomech., 17: 349-361.

DOI: 10.1016/0021-9290(84)90029-0

Aversa, R., D. Apicella, L. Perillo, R. Sorrentino and F. Zarone et al., 2009. Non-linear elastic threedimensional finite element analysis on the effect of endocrown material rigidity on alveolar bone remodeling process. Dental Mater., 25: 678-690. DOI: $10.1016 /$ j.dental.2008.10.015 
Aversa, R., F.I.T. Petrescu, R.V. Petrescu and A. Apicella, 2016. Biomimetic FEA bone modeling for customized hybrid biological prostheses development. Am. J. Applied Sci., 13: 1060-1067. DOI: 10.3844/ajassp.2016.1060.1067

Beaupre, G.S. and W.C. Hayes, 1985. Finite element analysis of a three dimensional open-celled model for trabecular bone. J. Biomech. Eng., 107: 249-56. PMID: 4046566

Bruno, R.J., P.A. Sauer, A.G. Rosenberg, J. Block and D.R. Sumner, 1999. The pattern of bone mineral density in the proximal femur and radiographic signs of early joint degeneration. J. Rheumatol., 26: 636-640.

Dalstra, M., R. Huiskes, A. Odgaard and V.L. Erning, 1993. Mechanical and textural properties of pelvic trabecular bone. J. Biomech., 26:523-535. DOI: 10.1016/0021-9290(93)90014-6

Frost, H.M., 1994. Wolff's law and bone's structural adaptations to mechanical usage: An overview for clinicians. Angle Orthod., 64: 175-188. DOI:10.1043/00033219(1994)064<0175:WLABSA $>2.0 . \mathrm{CO} ; 2$

Gramanzini, M., S. Gargiulo, F. Zarone, R. Megna and A. Apicella et al., 2016. Combined microcomputed tomography, biomechanical and histomorphometric analysis of the peri-implant bone: A pilot study in minipig model. Dental Mater., 32: 794-806. DOI: 10.1016/j.dental.2016.03.025

Lovejoy, C.O., 2005. The natural history of human gait and posture. Part 2. Hip and thigh. Gait Posture, 21: 113-24. DOI: 10.1016/j.gaitpost.2004.06.010

Lovejoy, C.O., 1988. Evolution of human walking. Sci. Am., 259: 118-25.

Lovejoy, C.O., R.S. Meindl, J.C. Ohman, K.G. Heiple and T.D. White, 2002. The Maka femur and its bearing on the antiquity of human walking: Applying contemporary concepts of morphogenesis to the human fossil record. Am. J. Phys. Anthropol., 119: 97-133.

Oh, I. and W.H. Harris, 1978. Proximal strain distribution in the loaded femur. An in vitro comparison of the distributions in the intact femur and after insertion of different hip-replacement femoral components. J. Bone Joint Surgery, 60: 75-85.

Kummer, B., 1986. Biomechanical principles of the statistics of the hip joint: A critical appraisal of a new theory. Zeitschrift fur Orthopadie und Ihre Grenzgebiete, 124: 179-187.

Perillo, L., R. Sorrentino, D. Apicella, A. Quaranta and E. Gherlone et al., 2010. Nonlinear visco-elastic finite element analysis of porcelain veneers: A submodelling approach to strain and stress distributions in adhesive and resin cement. J. Adhesive Dentistry, 12: 403-413.
Reilly, D.T. and A.H. Burstein, 1974. The mechanical properties of cortical bone. J. Bone Joint Surg., 56: 1001-1022.

Reilly, D.T. and A.H. Burstein, 1975. The elastic and ultimate properties of compact bone tissue. J. Biomech., 8: 393-369. DOI: $10.1016 / 0021-9290(75) 90075-5$

Schiraldi, C., D.A. Agostino, A. Oliva, F. Flamma and D.A. Rosa et al., 2004. Development of hybrid materials based on hydroxyethylmethacrylate as supports for improving cell adhesion and proliferation. Biomaterials, 25: 3645-3653. DOI: $10.1016 /$ j.biomaterials.2003.10.059

Sims, N.A. and T.J. Martin 2014. Coupling the activities of bone formation and resorption: A multitude of signals within the basic multicellular unit. BoneKEy Reports 3.

Sorrentino, R., D. Apicella, C. Riccio, E. Gherlone and F. Zarone et al., 2009. Nonlinear Visco-elastic finite element analysis of different porcelain veneers configuration. J. Biomed. Mater. Res. Part B Applied Biomater., 91: 727-736.

DOI: 10.1002/jbm.b.31449

Sorrentino, R., R. Aversa, V. Ferro, T. Auriemma and F. Zarone et al., 2007. Three-dimensional finite element analysis of strain and stress distributions in endodontically treated maxillary central incisors restored with diferent post, core and crown materials. Dent Mater., 23: 983-993.

DOI: $10.1016 /$ j.dental.2006.08.006

Tamar, G. and Z. Hashin, 1980. Analysis of viscoelastic behaviour of bones on the basis of microstructure. J. Biomech., 13: 89-96. DOI: 10.1016/0021-9290(80)90182-7

Walker, R.A. and C.O. Lovejoy, 1985. Radiographic changes in the clavicle and proximal femur and their use in the determination of skeletal age at death. Am. J. Phys. Anthropol., 68: 67-78.

Weinans, H., R. Huiskes and H.J. Grootenboer, 1992. The behavior of adaptive bone-remodeling simulation models. J. Biomech., 25: 1425-1441. DOI: 10.1016/0021-9290(92)90056-7 